\title{
The ANALGOR Online Inventory for Calibrating Analogical and Boolean Thinking in Normal Individuals
}

\author{
Desmond D. Mascarenhas \\ Mayflower Organization for Research and Education, Sunnyvale, CA, USA \\ Email: desmond2@mayflowerworld.org
}

How to cite this paper: Mascarenhas, D. D. (2019). The ANALGOR Online Inventory for Calibrating Analogical and Boolean Thinking in Normal Individuals. Psychology, 10, 1269-1274.

https://doi.org/10.4236/psych.2019.109082

Received: May 28, 2019

Accepted: July 8, 2019

Published: July 11, 2019

Copyright $\odot 2019$ by author(s) and Scientific Research Publishing Inc. This work is licensed under the Creative Commons Attribution International License (CC BY 4.0).

http://creativecommons.org/licenses/by/4.0/

\begin{abstract}
Abstractive functionality in the prefrontal cortex (PFC) using pattern-matching is believed to involve circuitry associated with the left inferior frontal gyrus whereas abstractions based on Boolean logic require the medial frontal cortex. How preferences and aptitudes for these distinct mechanisms vary amongst clinically normal individuals is not well understood. The possible influence of cultural environments on such aptitudes has also not been extensively investigated. In this initial study, a cohort $(\mathrm{n}=343)$ of English-speaking individuals from countries located on four continents (United States, United Kingdom, Australia, India) answered a fifteen-item ANALGOR survey, an online instrument designed to measure analogical and Boolean abstraction skills. Significant differences were observed between US scores on the one hand, and Australian and Indian scores on the other for analogical but not for Boolean abstractions. These initial observations await confirmation with larger cohorts.
\end{abstract}

\section{Keywords}

ANALGOR, Analogical, Metaphor, Theory-of-Mind, Abstraction, Boolean

\section{Background}

\subsection{Brain Regions Associated with Analogical and Boolean Logical Abstractions}

In experimental settings, the requirement to remap rules on to novel features recruits the left inferior rostrolateral prefrontal cortex and the lateral occipital complex (Hampshire, Thompson, Duncan, \& Owen, 2011). Abstract analogies, 
such as higher order metaphors-but not literal sentences-also activate left hemispheric regions such as the left inferior temporal cortex and the left lateral temporal lobe (Chen, Widick, \& Chatterjee, 2008). Conversely, abstract action rules subserved by Boolean logic appear to be dependent on circuits in the frontal cortex that are organized hierarchically along a rostrocaudal axis (Badre, Kayser, \& D'Esposito, 2010). Thus, different forms of abstraction critical to executive function appear to involve distinct regions of the human brain.

One universal element of analogical thinking is the implicit involvement of an abstraction step (Gentner \& Hoyos, 2017). The comprehension of high order metaphor, for example, involves "theory-of-mind" (ToM) abstraction in a way that literal meaning does not (Camp, 2006). Loss-of-function studies suggest that ToM functionality is largely dependent on abstractive construal and analogical mechanisms in the human brain (Baetens, Ma, Steen, \& Van Overwalle, 2014; Yuan \& Uttal, 2017). Conversely, abstract tasks associated with many other higher-order executive functions appear to employ Boolean logic.

\subsection{Reading-the-Mind-in-the-Eyes (REM) Test and the ANALGOR Inventory}

The Reading-of-the-Mind-in-the-Eyes (REM) Test has been widely used to measure the ability of subjects to recognize human emotions by looking at images of human eyes, a task dependent on ToM circuitry (Baron-Cohen, Jolliffe, Mortimore, \& Robertson, 1997). A revised REM test (Baron-Cohen, Wheelwright, Hill, Raste, \& Plumb, 2001), though improved from its original version, is nevertheless hampered by intrisic design issues, including the fact that it is often viewed by subjects as too long (subjects frequently fail to complete the test) and the ambiguity between target and foil words cited by the original authors leads to $>20 \%$ incorrect assignment to at least one foil word for nearly a third of the 40 items in the inventory. Moreover, the range of scores obtained with this test may not discriminate sufficiently between normal subjects (Olderbak, Wilhelm, Olaru, Geiger, Brenneman, \& Roberts, 2015). After removal of some ambiguous items, a slimmed-down version of the REM was successfully used to measure the effect of stress on analogical thinking in a normal female cohort (Mascarenhas, 2016) but even using this improved version, factor analysis of the dataset showed that only some of the original items showed adequate discriminant validity for the construct (data not shown). In the current work, these items were added to items designed to measure abstract reasoning requiring Boolean logic. The resulting hybrid inventory was dubbed ANALGOR (analogical-algorithmic) and was found to be of acceptable length to both male and female adult online respondents in cohorts from four different continents.

\section{Methods}

\subsection{Participants}

343 anonymous online participants were recruited from four countries (United 
States, United Kingdom, Australia, India) using the Survey Monkey Audience mechanism, which recruits and collects informed consent and demographic data from subjects worldwide. Additional information on this comprehensive mechanism may be found at surveymonkey.com. Informed consent was independently obtained again from each subject as part of the process prior to each survey in this study. Cohorts were balanced for age and income range as part of the Survey Monkey Audience specifications. The protocol for this study received approval from the Institutional Committee for Ethical Conduct in Research. The objective of the experimental design was to represent four different continents by selecting English-speaking participants from these four countries.

\subsection{Procedure}

Each participant completed the ANALGOR inventory online. Responses were collected over a two-week period in March-April 2019. All responses used in this study passed a software screen for fraudulent response patterns.

\subsection{ANALGOR Inventory}

In a recent study, nine items in the shortened REM inventory were found by factor analysis to show low ambiguity and high discriminant validity ([11]; see introduction section, above). These nine items (subscale A) were added to six items designed to measure Boolean abstract logic (subscale B) to create the ANALGOR inventory. Reliability (Cronbach's alpha) and discriminant validity for the two subscales of the ANALGOR were calculated using a multinational cohort in the current study, and found to be satisfactory.

\subsection{Statistical Analysis}

Data are presented as mean \pm SD. Probability values ( $p$ values) were computed using Student's $t$-test in Microsoft Excel. Pearson correlations were also computed using Excel. Cronbach's alpha was computed using Data Analysis tools from Real Statistics package incorporated into Excel as a free plugin downloaded from the Real Statistics website (http://www.real-statistics.com/).

\section{Results}

\subsection{Cronbach's Alpha}

Cronbach's alpha values calculated for the 9-item analogical abstraction sub-scale (subscale A) of the ANALGOR was 0.651, whereas for the 6-item Boolean abstraction sub-scale (subscale B) it was 0.452 . For both sub-scales, the deletion of any one component reduced the Cronbach's alpha.

\subsection{Discriminant Validity}

Pearson correlation between the two sub-scales of the ANALGOR was 0.19 (not statistically significant), supporting the discriminant validity of these two subscales. 


\subsection{Significant Differences between Cohorts}

Table 1 shows the ANALGOR scores of male and female subjects in each of the four continents represented in the cohort. Significant differences were seen between United States subjects and both Australian and Indian subjects in the analogical sub-scale of the ANALGOR but not between US and UK subjects. The relative preferences for analogical over Boolean abstract reasoning showed similar differences. These observations were independently seen in both male and female subjects from these geographic regions. With one exception (Indian females) no significant differences in Boolean abstract reasoning were seen between any of the geographic groups.

\section{Discussion}

\subsection{ANALGOR Scores}

The results reported here (Table 1) show a significant difference in analogical (subscale A) scores obtained from US and UK cohorts on the one hand, and Australian and Indian cohorts on the other. These differences were seen independently in both males and females. By contrast, only one geographic group (Indian females) differed significantly in their subscale B (abstract Boolean logic) scores.

Difference scores for the two subscales of the ANALGOR were computed for each subject by first converting scores under each subscale into $\mathrm{z}$ scores and then subtracting the B score from the A score. The average difference scores for cohorts varied significantly in exactly the same way the analogical score subscale scores did alone.

Table 1. Mean ANALGOR z scores for eight international cohorts. Mean \pm SD and p value (versus US cohort) are shown. Sample $\mathrm{n}$ and mean age of each cohort are also shown. "Preference" is a difference score i.e. the individually calculated difference between $\mathrm{z}$ scores for analogic and algorithmic subscales (see text). Top panel: male cohorts; bottom panel: female cohorts.

\begin{tabular}{|c|c|c|c|c|c|c|c|c|c|c|c|}
\hline \multirow{2}{*}{$\begin{array}{l}\text { MALES: } \\
\text { Region }\end{array}$} & \multirow{2}{*}{ (n) } & \multirow{2}{*}{ Mean Age } & \multicolumn{3}{|c|}{ ANALOGICAL } & \multicolumn{3}{|c|}{ ALGORITHMIC } & \multicolumn{3}{|c|}{ PREFERENCE } \\
\hline & & & Mean & $\mathrm{SD}$ & $p$ & Mean & SD & $p$ & Mean & $\mathrm{SD}$ & $p$ \\
\hline United States & 40 & $43.6 \pm 16.7$ & 0.519 & 0.709 & & 0.250 & 1.095 & & 0.211 & 0.964 & \\
\hline Australia & 23 & $43.7 \pm 9.1$ & -0.247 & 0.871 & 0.001 & 0.122 & 1.082 & 0.655 & -0.290 & 0.856 & 0.038 \\
\hline UK & 26 & $43.4 \pm 18.5$ & 0.208 & 0.837 & 0.124 & -0.118 & 0.874 & 0.065 & 0.327 & 0.891 & 0.410 \\
\hline India & 60 & $43.1 \pm 4.9$ & -0.557 & 0.958 & 0.000 & 0.027 & 1.085 & 0.319 & -0.458 & 1.042 & 0.001 \\
\hline Total (n) & 149 & & & & & & & & & & \\
\hline \multirow{2}{*}{$\begin{array}{l}\text { FEMALES: } \\
\text { Region }\end{array}$} & \multirow{2}{*}{ (n) } & \multirow{2}{*}{ Mean Age } & \multicolumn{3}{|c|}{ ANALOGICAL } & \multicolumn{3}{|c|}{ ALGORITHMIC } & \multicolumn{3}{|c|}{ PREFERENCE } \\
\hline & & & Mean & SD & $p$ & Mean & SD & $p$ & Mean & SD & $p$ \\
\hline United States & 98 & $40.1 \pm 15.1$ & 0.605 & 0.713 & & 0.220 & 1.035 & & 0.302 & 0.881 & \\
\hline Australia & 27 & $40.3 \pm 7.8$ & 0.078 & 1.082 & 0.023 & 0.211 & 0.975 & 0.965 & -0.104 & 0.855 & 0.035 \\
\hline UK & 17 & $45.0 \pm 9.3$ & 0.571 & 0.859 & 0.881 & 0.345 & 0.836 & 0.590 & 0.178 & 0.814 & 0.572 \\
\hline India & 52 & $43.7 \pm 7.7$ & -0.565 & 0.944 & 0.000 & -0.324 & 1.013 & 0.002 & -0.189 & 1.012 & 0.004 \\
\hline Total (n) & 194 & & & & & & & & & & \\
\hline
\end{tabular}




\subsection{Implications and Limitations}

This is a preliminary finding in a small cohort. A larger study will be needed to confirm these initial observations. The practical implications of this finding, if confirmed by future studies, are potentially significant in the management of a knowledge economy within which shorter product cycles has created a growing dependence on innovation (Steel, Rinne, \& Fairweather, 2012; Mascarenhas \& Singh, 2012; Mascarenhas \& Vander Veer, 2014; Mascarenhas, 2017). Analogical thinking has long been associated with creative thinking and innovation. However, a threshhold level of general intelligence involving frontal cortex circuitry is also thought to be a requirement for innovative thinking (Weinberger, Iyer, \& Green, 2016).

The REM utilizes photographs of Caucasian subjects and descriptive single-word adjectives for emotions, thereby minimizing but not eliminating its reliance on Eurocentric language and culture. It is possible that the Indian cohort was disproportionately affected by these residual cultural and language variables. Although all participants from all countries were nominally English-speaking, we did not establish the proportion of subjects that spoke English as a first language. This is a limitation in the current experiment and would need to be addressed in future studies. Nevertheless, these concerns would not explain the significant differences observed between US and Australian cohorts.

In summary, the data from this study show significant differences in analogical (but not Boolean) abstraction performance in subjects recruited from four countries located on four different continents.

\section{Conflicts of Interest}

The author declares no conflicts of interest regarding the publication of this paper.

\section{References}

Badre, D., Kayser, A. S., \& D’Esposito, M. (2010). Frontal Cortex and the Discovery of Abstract Action Rules. Neuron, 66, 315-326.

https://www.ncbi.nlm.nih.gov/pubmed/?term=20435006

https://doi.org/10.1016/j.neuron.2010.03.025

Baetens, K., Ma, N., Steen, J., \& Van Overwalle, F. (2014). Involvement of the Mentalizing Network in Social and Non-Social High Construal. Social Cognitive Affect Neuroscience, 9, 817-824. https://www.ncbi.nlm.nih.gov/pubmed/23552077

https://doi.org/10.1093/scan/nst048

Baron-Cohen, S., Jolliffe, T., Mortimore, C., \& Robertson, M. (1997). Another Advanced Test of Theory of Mind: Evidence from Very High Functioning Adults with Autism or Asperger Syndrome. Journal of Child Psychology and Psychiatry, 38, 813-822.

https://www.ncbi.nlm.nih.gov/pubmed/?term $=9363580$

https://doi.org/10.1111/j.1469-7610.1997.tb01599.x

Baron-Cohen, S., Wheelwright, S., Hill, J., Raste, Y., \& Plumb, I. (2001). The "Reading the Mind in the Eyes" Test Revised Version: A Study with Normal Adults, and Adults with Asperger Syndrome or High-Functioning Autism. Journal of Child Psychology and 
Psychiatry, 42, 241-251. https://www.ncbi.nlm.nih.gov/pubmed/?term=11280420 https://doi.org/10.1111/1469-7610.00715

Camp, E. (2006). Metaphor in the Mind: The Cognition of Metaphor. Philosophy Compass, 1, 154-170.

https://onlinelibrary.wiley.com/doi/abs/10.1111/j.1747-9991.2006.00013.x

https://doi.org/10.1111/j.1747-9991.2006.00013.x

Chen, E., Widick, P., \& Chatterjee, A. (2008). Functional-Anatomical Organization of Predicate Metaphor Processing. Brain and Language, 107, 194-202.

https://www.ncbi.nlm.nih.gov/pubmed/18692890 https://doi.org/10.1016/j.bandl.2008.06.007

Gentner, D., \& Hoyos, C. (2017). Analogy and Abstraction. Topics in Cognitive Science, 9, 672-693. https://www.ncbi.nlm.nih.gov/pubmed/?term=28621480 https://doi.org/10.1111/tops.12278

Hampshire, A., Thompson, R., Duncan, J., \& Owen, A. M. (2011). Lateral Prefrontal Cortex Subregions Make Dissociable Contributions during Fluid Reasoning. Cereb Cortex, 21, 1-10. https://www.ncbi.nlm.nih.gov/pubmed/?term=20483908 https://doi.org/10.1093/cercor/bhq085

Mascarenhas, D. D. (2016). Association of Chronic Stress with Agency, Theory of Mind Function and Abstract Construal in Women. Psychology, 7, 1397-1401.

http://file.scirp.org/Html/2-6901934_71749.htm

https://doi.org/10.4236/psych.2016.712140

Mascarenhas, D. D. (2017). Stress, Brain Wiring and the Economy. Journal of Psychology and Psychotherapy, 7, 318.

https://www.longdom.org/open-access/stress-brain-wiring-and-the-economy-2161-048 7-1000318.pdf

Mascarenhas, D. D., \& Vander Veer, S. (2014). Women, Innovation and Literature. Journal of Innovation and Entrepreneurship, 3, 7.

https://link.springer.com/article/10.1186/2192-5372-3-7 https://doi.org/10.1186/2192-5372-3-7

Mascarenhas, D., \& Singh, A. H. (2012). Regional Culture and Adaptive Behavior of Physicians. Journal of Bioeconomics, 14, 257-266.

https://link.springer.com/article/10.1007/s10818-011-9115-z

https://doi.org/10.1007/s10818-011-9115-z

Olderbak, S., Wilhelm, O., Olaru, G., Geiger, M., Brenneman, M. W., \& Roberts, R. D. (2015). A Psychometric Analysis of the Reading the Mind in the Eyes Test: Toward a Brief form for Research and Applied Settings. Frontiers in Psychology, 6, 1503-1508. https://www.ncbi.nlm.nih.gov/pubmed/26500578 https://doi.org/10.3389/fpsyg.2015.01503

Steel, G. D., Rinne, T., \& Fairweather, J. (2012) Personality, Nations, and Innovation: Relationships between Personality Traits and National Innovation Scores. Cross-Cultural Research, 46, 3-8. https://doi.org/10.1177/1069397111409124

Weinberger, A. B., Iyer, H., \& Green, A. E. (2016). Conscious Augmentation of Creative State Enhances "Real" Creativity in Open-Ended Analogical Reasoning. PLoS ONE, 11, e0150773. https://www.ncbi.nlm.nih.gov/pubmed/?term=26959821 https://doi.org/10.1371/journal.pone.0150773

Yuan, L., \& Uttal, D. H. (2017). Analogy Lays the Foundation for Two Crucial Aspects of Symbolic Development: Intention and Correspondence. Topics in Cognitive Science, 9, 738-757. https://www.ncbi.nlm.nih.gov/pubmed/?term=28481471 https://doi.org/10.1111/tops.12273 